\title{
Sugar free medicines-are you using them?
}

Tooth decay is becoming less prevalent among British schoolchildren. The proportion of 5 year olds in England and Wales with recognised dental decay decreased from $71 \%$ in 1973 to $48 \%$ in $1983 .{ }^{1}$ Public awareness. improved diet, falling confectionary sales, dental hygiene, and fluoridation may all be contributing to this long awaited improvement in the health of the nation's teeth. Currently, more than half of the children aged 5 have visited a dentist but have not needed extractions or fillings. This would seem to endorse the view that the dental profession is playing a major and not necessarily invasive role in promoting dental health. Nevertheless, dentists have recently been subjected to critical review by monitoring bodies, notably the Consumers' Association. ${ }^{2}$ The growing number of children with little or no dental caries are advised not to visit their dentist too regularly in case they are subjected to unnecessary fillings. It is perhaps surprising that the critical spotlight has not yet been brought to bear on the cariogenic prescribing habits of doctors. Parents, proud of their brood's unblemished dentition and conditioned by years of disciplining eating habits, can surely expect doctors to help them protect their investment. Long term prescriptions of sugar containing medicines can no longer be tolerated when alternatives are available or can be made available.

The paediatrician's inspection of a child's teeth is often perfunctory, a superficial review as he focuses on the more familiar territory of tongue, palate, and pharynx. We rest assured in the knowledge that we abandoned tetracyclines decades ago, and we frown on contentment induced by a blackcurrant juice loaded bottle or a dummy dipped in the honey pot. How many of us consider the sugar content of the elixirs, linctuses, and mixtures that we so regularly prescribe? Too often they contain sucrose, glucose, or fructose in a tenacious liquid ideal for promoting the accumulation of plaque, the extracellular polysaccharide gelatinous layer that provides a protected environment for oral streptococci. These organisms rapidly ferment sugar to acid, which in turn decalcifies enamel and lays bare dentine. This acid attack is rapid and less amenable to prompt teeth brushing than was previously believed.

Children's teeth are unlikely to be harmed by occasional brief courses of sugar containing medicines. Asthma, convulsions, and recurrent urinary tract infections, however, are examples of common disorders that provide a sizable number of children liable to be exposed to long term treatment with drugs. As a general rule, paediatricians are responsible for either beginning such treatment or for setting local standards for family practitioner guidance. Faced with the challenge of promoting compliance in a culture that has grown to demand that all tastes be inoffensive, paediatricians have passively promoted the manufacture and use of sucrose containing medicines. Only relatively recently has the topic been explored in the medical press. Research into deleterious effects of sugar based medicines has not been popular. It is salutary to contrast the detailed, and no doubt fund attracting, research focused on the mechanisms by which anticonvulsants marginally modify vitamin $\mathrm{D}$ metabolism with the dearth of studies on the more conspicuous harm done to teeth by the sugar accompanying the anticonvulsants. In spite of this 'Cinderella status', at least one well documented study has confirmed that children taking syrup medicines for six months or longer had significantly more carious teeth, gingivitis, and peridontal disease than healthy children matched for age, diet, tooth brushing habit, and socioeconomic state. ${ }^{3}$ In other words, iatrogenic dental disease and all that it implies in terms of pain, anxiety, time, and cost is being added to the morbidity of already disadvantaged children.

The campaign against sugar in medicines is gaining momentum. Spearheaded by the Pharmaceutical Society and the British Dental Association, it is consulting with manufacturers to accelerate the reformulation of liquid medicines. Alternative sweeteners such as sorbitol and xylotol are now appearing in more preparations. The highly competitive bronchodilator market has recently illustrated how the avoidance of sucrose provides an advertising advantage. A major obstacle to noncariogenic prescribing lies within the pages of the British National Formulary ${ }^{4}$ and relates to the diluent directory. Contracted retail chemists, but not hospital pharmacists, are instructed that 'when a prescription for a liquid oral preparation is written and the dose ordered is less than $5 \mathrm{ml}$, the preparation will normally be diluted with a suitable vehicle so that the required dose is contained in 5 ml'. The recommended vehicle is usually syrup based, introducing instability as well as potential harm to teeth. The Licensing Authority has yet to respond to pressure from pharmacists and the Drug 
and Therapeutics Bulletin. ${ }^{5}$ The Authority could also help manufacturers by revising instructions and guidelines for the introduction of improved formulations. Possibly the editors of the British National Formulary might consider the introduction of a S/F symbol to denote sugar free preparations. In the meantime doctors can make greater use of tablets crushed and taken with a sugarless drink. There are precedents for infants and young children coping admirably with tablets.

Paediatricians are justifiably cautious in changing their prescribing habits. The time is overdue, however, for rallying to the support of the campaign for sugar free medicines. Comprehensive lists are available from drug information centres (for example, the North West Drug Information Centre's leaflet 'Sugar content of paediatric medicines') and should be stapled to copies of the British National Formulary used in children's departments. Drug representatives whose paediatric formulations fail to appear on the list might be invited to return only when they do.

\section{References}

1 In: Todd JE, Dodd T, eds. Children's dental health in the United Kingdom 1983. HMSO: London, 1985.

2 Anonymous. Routine six-monthly checks for dental disease? [Editorial]. Drug Ther Bull 1985;23:69-72.

3 Roberts IF, Roberts GJ. Relationship between medicines sweetened with sucrose and dental disease. Br Med J 1979;2: 14-16.

4 Joint Formulary Committee. Prescribing for children. In: British National Formulary. Vol. 10. London: British Medical Association and The Pharmaceutical Society of Great Britain, 1985:6.

5 Anonymous. Liquid medicines can rot sick children's teeth [Editorial]. Drug Ther Bull 1981;19:75-6.

D I JOHNSTON

Children's Department, University Hospital, Queen's Medical Centre, Nottingham 